\title{
Photochemistry and photophysics of cholesta-5,7,9(11)-trien-3ß-ol: a fluorescent analogue of cholesterol
}

\author{
Jack Saltiel ${ }^{1}$ (D) - Sumesh B. Krishnan ${ }^{1}$. Shipra Gupta ${ }^{1}$ - E. Aliea Hernberg ${ }^{1} \cdot$ Ronald J. Clark $^{1}$
}

Received: 4 September 2021 / Accepted: 1 November 2021 / Published online: 9 January 2022

(c) The Author(s), under exclusive licence to European Photochemistry Association, European Society for Photobiology 2021

\begin{abstract}
Cholesta-5,7,9(11)-trien-3 $\beta$-ol (9,11-dehydroprovitamin $\left.\mathrm{D}_{3}, \mathrm{CTL}\right)$ is used as a fluorescent probe to track the presence and migration of cholesterol in vivo. CTL is known to be photochemically active, but little consideration has been given to the formation efficiency and possible toxicity of its photoproducts. In degassed tetrahydrofuran (THF) solution, we isolated the photoproduct of CTL and of its 25-hydroxy derivative (HOCTL), and X-ray crystal structures were obtained for HOCTL and the photorearrangement product. The X-ray crystal structure and its ${ }^{1} \mathrm{H}$ NMR spectrum confirm the product structure as a pentacyclic HOCTL isomer. In the presence of air in THF, endoperoxide formation via [2+4] addition of ${ }^{1} \mathrm{O}_{2}{ }^{*}$ across the $\mathrm{B}$ ring of CTL or HOCTL becomes the dominant photoreaction. The UV spectrum and decay kinetics of the triplet state of HOCTL, the precursor of ${ }^{1} \mathrm{O}_{2}{ }^{*}$, are determined by transient absorption spectroscopy. We confirm the proposed structure of the endoperoxide by $\mathrm{X}$-ray crystallography. Kinetics analysis of quantum yields provides rate constants for photophysical and photochemical events.
\end{abstract}

\section{Graphical abstract}

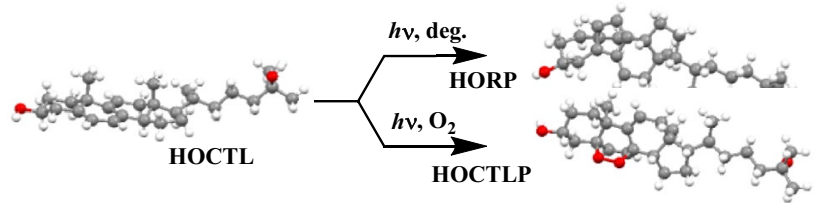

Keywords Cholestatrienol rearrangement $\cdot$ Endoperoxide phototoproducts $\cdot$ Fluorescence triplet yields

\section{Introduction}

The natural products cholesta-5,7,9(11)-trien-3 $\beta$-ol (CTL) and its close relative 9(11)-dehydroergosterol (DHE) differ from provitamin $\mathrm{D}_{3}$ (Pro) and ergosterol (E), respectively, by the additional $\mathrm{C}_{9}-\mathrm{C}_{11}$ double bond [1-6]. The cholestane skeleton lends rigidity to their embedded conjugated triene moieties increasing $S_{1}$ lifetimes by diminishing torsional relaxation, thereby rendering them fluorescent [7]. Their structural similarity to cholesterol, Chart 1 , accounts for their use as ideal fluorescence probes for studies of cholesterol trafficking and membrane organization in vivo [8-11]. Their

Jack Saltiel

jsaltiel@fsu.edu

1 Department of Chemistry and Biochemistry, Florida State University, Tallahassee, FL 32306-4390, USA fluorescence intensity is diminished on prolonged excitation leading to photobleaching and fluorescence recovery studies [12]. The photoproducts obtained on direct excitation of CTL and DHE in vitro were investigated in pioneering studies by the Windaus and Barton groups [13-15]. We determine the correct diastereomer of the structure, Scheme 1, proposed by Barton and Kende for the photoproduct [15]. Windaus and Linsert also reported the eosin photosensitized oxidation of DHE in ethanol [13]. The accepted structure of the photooxidation product (DHEP, $5 \alpha, 8 \alpha$-epidioxy-22-Eergosta-6,9(11),22-trien-3 $\beta$-ol) was proposed by Fieser [16], before the role of ${ }^{1} \Delta_{\mathrm{g}}$ singlet oxygen $\left({ }^{1} \mathrm{O}_{2}{ }^{*}\right)$ in such reactions was known. ${ }^{1} \mathrm{O}_{2}{ }^{*}$ luminescence observed on irradiation of an air-saturated solution of CTL in $\mathrm{CCl}_{4}$ established formation of CTL triplets that transfer energy to ${ }^{3} \mathrm{O}_{2}$ [15]. It was suggested that CTL, naturally present in skin, may contribute to skin photoaging and UV-induced skin cancer by sensitizing 
the formation of ${ }^{1} \mathrm{O}_{2} *$ [17]. Furthermore, hydroperoxide formation via the ${ }^{1} \mathrm{O}_{2}{ }^{*}$ oxidation of ergosterol may be a route to DHE [18]. The peroxides of CTL and DHE, CTLP, and DHEP, respectively, are also known natural products [5, 19]. Both ergosterol peroxide, EP, and DHEP have been shown to be cytotoxic [19].

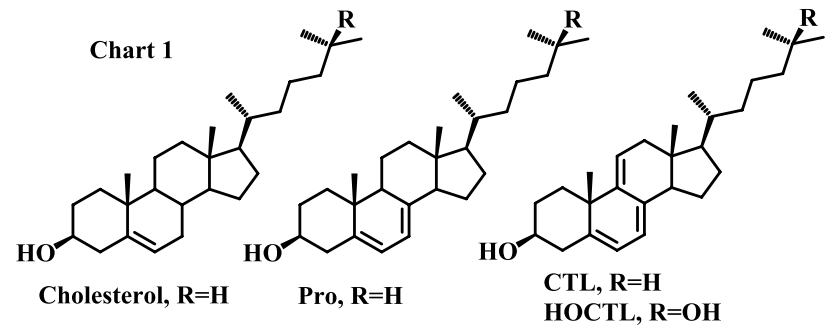

In view of their wide use as fluorescence probes, there has been surprisingly little consideration of the formation efficiency and possible toxicity of CTL and DHE photoproducts. In this paper, we identify photoproducts formed following direct excitation of CTL and HOCTL in degassed and air-saturated THF solutions. A 1930 study that reported the UV evolution of the photochemical loss of DHE in the course of its direct excitation in ethanol [20] found the $\lambda_{\max }$ of the photoproduct mixture at $243 \mathrm{~nm}$ instead of at $261 \mathrm{~nm}$, as expected for the major isolated photoproduct [13-15]. The participation of ethanol in the photoreactions of CTL and HOCTL leads to several additional photoproducts and will be addressed separately [21]. We show here that on irradiation in degassed THF, HOCTL quantitatively rearranges by $9,10 \sigma$-bond cross-addition to the $5,6 \pi$-bond to give the pentacyclic isomer $(3 R, 3 \mathrm{a} R, 5 \mathrm{~b} S, 7 S, 9 \mathrm{a} S, 9 \mathrm{~b} S)$ 3-((R)-6-hydroxy-6-methylheptan-2-yl)-3a,9a-dimethyl1,2,3,3a, 4, 6, 7, 8,9,9a,9b,10b-dodecahydrobenzo[2,3] cyclopropa[1,2-b]-as-indacen-7-ol (HORP, $\lambda_{\max }=261 \mathrm{~nm}$ ). In the presence of air, at sufficiently high [CTL], peroxide formation dominates. The $25-\mathrm{OH}$ substituent does not influence the photochemistry or the photophysics, Scheme 1.

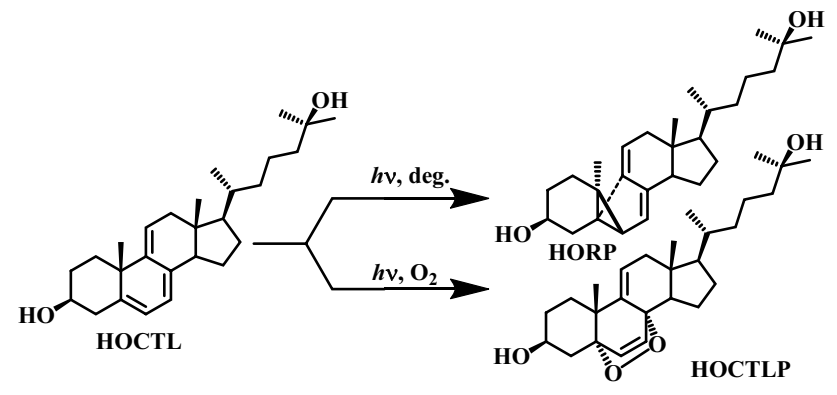

Scheme 1 Photochemistry of HOCTL in THF

\section{Experimental section}

\subsection{Materials}

Unless otherwise stated, reagent grade solvents from commercial sources were used as received. We recrystallized 7-dehydrocholesterol (provitamin $\mathrm{D}_{3}$, Pro, US Biochemical Corp.) from ethanol. 25-Hydroxyprovitamin $\mathrm{D}_{3}$ (Isogen, 96.5\%) was recrystallized twice from methanol and twice from a 3/1 ethyl acetate/hexane mixture. Alternatively, purification was by column chromatography on silica. For spectroscopic measurements, purification was by HPLC. Tetrahydrofuran (THF, Sigma-Aldrich, ACS reagent, 99\%, containing $250 \mathrm{ppm}$ BHT as inhibitor) was freshly distilled prior to use. Ethanol (Pharmco-AAPER) was 200 proof, absolute/anhydrous. $\mathrm{CHCl}_{3}$ (Aldrich) and glacial acetic acid (Fisher) were used as received. $\mathrm{Hg}(\mathrm{OAc})_{2}$ (Acros) and benzophenone (Fisher Scientific, 99.69\% GC purity) were used as received.

\subsection{Analytical methods}

Analysis of product compositions was done by GC, by ${ }^{1} \mathrm{H}$ NMR, by UV-Vis, by fluorescence, by MS, and by GC/ MS. We used a Bruker/Varian CP-3800 gas chromatograph equipped with an electronic integrator for CTL and for stilbene actinometry. Semi-preparative HPLC was performed using a Beckman Coulter System Gold 125 solvent module with a System Gold 168 detector. UV-Vis absorption spectra were measured on a Varian Cary 300B Spectrometer. Fluorescence spectra were measured using a Horiba Fluoromax 4 fluorometer. Fluorescence lifetimes were determined using a different Horiba Fluoromax 4 instrument equipped with a time-correlated single-photon counting accessory and an R928 PMT detector (Hamamatsu). The light source was a $296 \mathrm{~nm}$ nanoLED (Horiba) having a pulse duration of $<0.75 \mathrm{~ns}$ and $1 \mathrm{MHz}$ repetition rate. Photon count was set at 10,000 and the time-to-amplitude converter range was set at $50 \mathrm{~ns}$. The instrument response function was obtained by collecting Rayleigh scatter at $296 \mathrm{~nm}$. Fluorescence lifetimes were determined by reconvoluting the instrument response function with exponential decay using DAS6 (Horiba) fluorescence decay analysis software. The quality of the fits was judged by $\chi^{2}$ values, standard deviations of derived lifetimes, and visual inspection of the residuals. Absolute fluorescence quantum yields were measured with the use of a Hamamatsu Quantaurus-QY Spectrometer equipped with a $150 \mathrm{~W}$ Xenon arc lamp. The instrument employs an integrated sphere sample chamber with a cooled back-thinned 1024 channel charge coupled device sensor as detector. It avoids the need for a fluorescence standard using the attenuation in the area 
of the Rayleigh scattered light peak at $\lambda_{\text {exc }}=325 \mathrm{~nm}$ to measure photon absorption. HOCTL solutions were outgassed with oxygen or argon for $2 \mathrm{~h}$ prior to quantum yield and lifetime measurements. Transient absorption measurements and triplet decay kinetics were measured using an Edinburgh Instruments LP980-KS Laser Flash Photolysis Spectrometer. A continuum Nd:YAG laser provided the excitation pulse and transient absorption was monitored with a $150 \mathrm{~W}$ Xenon lamp. Brüker $500 \mathrm{MHz}$ NMR spectrometer was used to measure NMR spectra in $\mathrm{CDCl}_{3}$. We used a Bruker Autoflex III MALDI TOF Mass Spectrometer for matrix-assisted laser desorption/ionization measurements.

\subsection{Crystallography}

HOCTL, HORP, and HOCTLP crystals were mounted on a nylon loop and centered in the beam of $0.71073 \AA$ Mo X-rays. After indexing, frame data were obtained on a Bruker SMART APEX 2 diffractometer at $103 \mathrm{~K}$ using a detector distance of $6 \mathrm{~cm}$. Frame collection times of 40 and $60 \mathrm{~s}$ were used in an effort to obtain data at higher angles. The data-to-parameter ratio was always more than 10:1. The integration was performed using the program SAINT, which is part of the Bruker suite of programs. An absorption correction was done by SADABS and the program XPREP used to determine the space group. The structures were solved by direct methods and refined by SHELXTL. The non-hydrogen atoms were refined anisotropically. The hydrogen atoms were assigned except for those on the oxygen atoms of the hydroxyl substituents and of water solvent molecules.

\subsection{Irradiation procedures}

Sample preparation and degassing procedures were described previously [22]. We used Hanovia reactors for preparative experiments and a Moses merry-go-round [23] apparatus, immersed in a thermostated water bath, for quantum yield measurements. We employed medium pressure 200-W and 450-W Hg lamps. The $313 \mathrm{~nm} \mathrm{Hg}$ line was isolated using a potassium perchromate/potassium carbonate filter solution [22]. The trans $\rightarrow$ cis photoisomerization of stilbene in pentane $\left(\lambda_{\mathrm{exc}}=313 \mathrm{~nm}\right)$ was used for actinometry, $\phi_{\mathrm{tc}}=0.52$ [22]. Pyrex tubes, $13 \mathrm{~mm}$ o.d., fitted with standard taper joints and grease traps were loaded with $3.0 \mathrm{~mL}$ aliquots of solutions. They were degassed using $4-6$ freeze-pump-thaw cycles to $<10^{-4}$ Torr and flame sealed at a constriction. All operations, including analyses, were performed under nearly complete darkness (red light). Ar, $\mathrm{N}_{2}$, and $\mathrm{O}_{2}$ outgassed solutions were used in some experiments.

\section{Results}

\subsection{Synthesis of CTL and HOCTL}

The dehydrogenation of ergosterol to DHE using mercuric acetate was first described by Windaus and Linsert [13] and the method was applied to the conversion of several $\Delta^{5,7}$-steroids, including Pro, to the corresponding $\Delta^{5,7,9}$ steroids by Antonucci et al. [24] We based our synthesis on the somewhat modified version described by Fischer et al. [7] The following procedure for the synthesis of CTL is typical: Pro $(2.0 \mathrm{~g}, 5.2 \mathrm{mmol})$ and $\mathrm{Hg}(\mathrm{OAc})_{2}$ $(3.0 \mathrm{~g}, 9.4 \mathrm{mmol})$ were added to $40 \mathrm{~mL}$ of $\mathrm{CHCl}_{3}$ in a three-necked $100 \mathrm{~mL}$ round-bottom flask equipped with a stirring bar and rubber septa at the three necks. Glacial acetic acid $(17 \mathrm{~mL})$ was added to the solution, and after the reaction mixture was outgassed with $\mathrm{N}_{2}$ using entry and exit syringe needles, it was placed in a thermostated oil bath. The reaction mixture was stirred under $\mathrm{N}_{2}$ at $40{ }^{\circ} \mathrm{C}$ for $18 \mathrm{~h}$. The progress of the reaction was followed by TLC (10\% ethyl acetate/hexanes). Precipitated mercury salts were removed by filtration and washed with $\mathrm{CHCl}_{3}$. The combined filtrate and washings were transferred to a $100 \mathrm{~mL}$ Erlenmeyer flask and neutralized using a saturated aqueous $\mathrm{NaHCO}_{3}$ solution. The organic layer was separated and the aqueous layer was washed $3 \times$ with $\mathrm{CHCl}_{3}$. After drying the combined organic solutions using anhydrous sodium sulfate, we removed the solvent under reduced pressure to obtain $1.54 \mathrm{~g}$ of a brown gummy material. This residue was purified by repeated column chromatographies on silica using progressively increasing ethyl acetate content in hexanes (up to $6 \%$, initially and up to $3 \%$ in the third chromatography) eluents were monitored by UV-Vis. The final yield was $400 \mathrm{mg}$ (20\%) of CTL. HOCTL was similarly obtained with the exception that, due to its greater polarity, the ethyl acetate eluent was replaced by ethanol. In numerous runs, yields of CTL and HOCTL never exceeded 30\%. The starting materials Pro and HOPro eluted very close to the CTL and HOCTL products and were exceedingly difficult to remove completely by column chromatography. This was accomplished for the final experiments by the use of semi-preparative HPLC. Residual HOPro in initial HOCTL samples did not interfere with fluorescence and most photochemical measurements, because its UV absorption is well to the blue of CTL (Fig. S1 in the Supporting Information, SI). For quantum yield and X-ray measurements, further purification of CTL and HOCTL was done by HPLC and by recrystallization. We obtained crystals of HOCTL suitable for X-ray diffraction analysis from $\mathrm{CH}_{3} \mathrm{CN}$ and benzene in 3:1 ratio with a drop of deionized $\mathrm{H}_{2} \mathrm{O}$. Figure 1 shows the $\mathrm{X}$-ray HOCTL structure. 


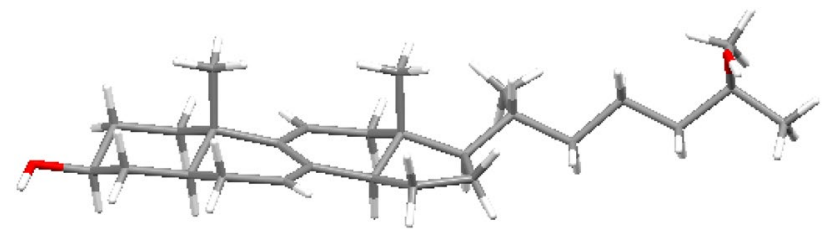

Fig. 1 The X-ray structure of HOCTL

Table $1 \mathrm{O}_{2}$ Quenching of HOCTL fluorescence in THF, $20^{\circ} \mathrm{C}$

\begin{tabular}{llllll}
\hline & $10^{3}\left[\mathrm{O}_{2}\right] / \mathrm{M}$ & $\phi_{\mathrm{f}}$ & $\tau_{\mathrm{f}} / \mathrm{ns}$ & $\chi^{2}$ & $10^{-8} \phi_{\mathrm{f}} / \tau_{\mathrm{f}}$ \\
\hline $\mathrm{Ar}$ & 0 & $0.033_{5}$ & $0.22_{5}$ & 1.24 & 1.49 \\
$\mathrm{Air}$ & 1.72 & $0.032_{4}$ & $0.22_{1}$ & 1.18 & 1.47 \\
$\mathrm{O}_{2}$ & 8.27 & $0.029_{2}$ & $0.20_{8}$ & 1.08 & 1.40 \\
\hline
\end{tabular}
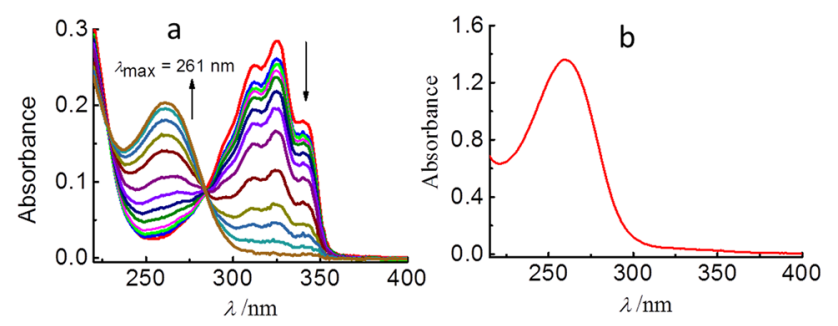

Fig. 2 Irradiation of $\mathbf{a}[\mathrm{HOCTL}]_{0}=5.23 \times 10^{-5} \mathrm{M}$ in THF; $\mathbf{b}$ the UV spectrum of pure HORP in THF

\subsection{Fluorescence measurements}

We determined the effects of $\mathrm{O}_{2}$ on HOCTL fluorescence quantum yield and lifetime by measuring the fluorescence of Ar-bubbled, air-saturated and $\mathrm{O}_{2}$-bubbled $1.05 \times 10^{-5} \mathrm{M}$ THF solutions at room $T\left(20.3^{\circ} \mathrm{C}\right)$, Table 1 . The oxygen and the argon-saturated HOCTL solutions were outgassed with $\mathrm{O}_{2}$ and $\mathrm{Ar}$ for $2 \mathrm{~h}$ prior to the quantum yield $\left(\lambda_{\text {exc }}=325 \mathrm{~nm}\right)$ ) and lifetime measurements. A pre-bubbler was employed to minimize solvent loss. For the lifetime measurements, samples were excited with a $295 \mathrm{~nm},<0.75 \mathrm{~ns}$ diode pulse, and fluorescence decay was monitored at $367 \mathrm{~nm}$. The contribution of a small ( $<3 \%)$ longer lived component, $\tau=11.9 \mathrm{~ns}$ under $\mathrm{Ar}$, diminishes and its lifetime decreases as the concentration of oxygen is increased. Lifetime and $\chi^{2}$ values are given in Table 1.

\subsection{Photochemical observations}

The degassed THF solution (six freeze-pump-thaw cycles to $<10^{-4}$ Torr and flame sealed at a constriction) was irradiated in a $13 \mathrm{~mm}$ Pyrex test tube provided with a sidearm to which a $0.50 \times 1.0 \mathrm{~cm}$ quartz cell was attached via a graded seal. Figure 2 shows the UV evolution of HOCTL
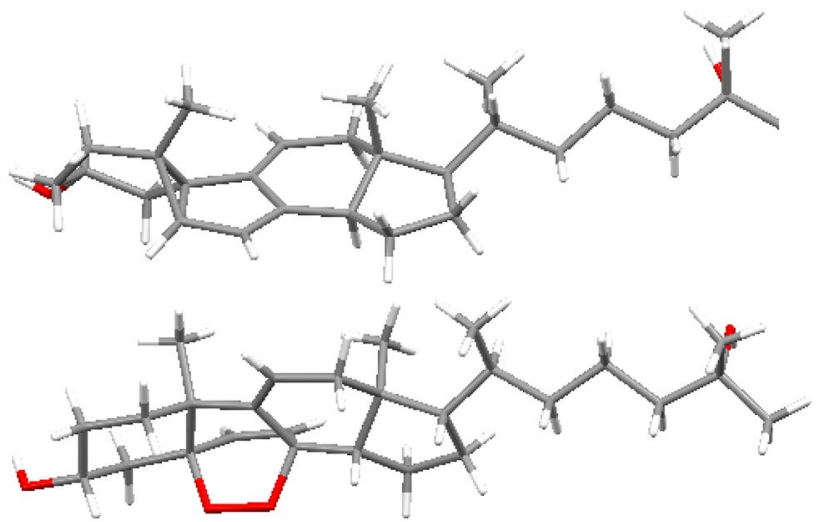

Fig. 3 X-ray crystal structures of HORP (upper) and HOCTLP

irradiated at $\lambda_{\text {exc }}=313 \mathrm{~nm}$ at $23.5^{\circ} \mathrm{C}$. The concentration given in the caption of Fig. 2 is based on our measured molar absorptivity coefficient of HOCTL at $\lambda_{\max }=324 \mathrm{~nm}$ $\left(1.1_{0} \times 10^{4} \mathrm{M}^{-1} \mathrm{~cm}^{-1}\right.$, Fig. S2 in SI). Figure S3 in SI gives the Beer-Lambert plot for HORP in THF.

The MALDI spectrum measured for CTL photoproducts in air-saturated THF shows $\mathrm{m} / \mathrm{z}$ peaks at 404.72 and 436.78 for rearrangement product $\mathrm{RP}$ (382 for CTL +23 for $\mathrm{Na}^{+}$) and peroxide product CTLP (382 for CTL +23 for $\mathrm{Na}^{+}+32$ for $2 \mathrm{xO}$ ), respectively, (Fig. S4 in SI). Together, the two spectra account for all product peaks in the ${ }^{1} \mathrm{H}$ NMR spectrum of the product mixture in degassed solutions. The ${ }^{1} \mathrm{H}$ NMR spectrum of RP $\left(\mathrm{CDCl}_{3}, 500 \mathrm{MHz}\right): 5.42(\mathrm{~s}, 1 \mathrm{H}) ; 5.35(\mathrm{dd}$, $1 \mathrm{H}, J=5.4 \mathrm{~Hz}$ ); 3.68 (“q", $1 \mathrm{H}, J=9.5 \mathrm{~Hz}) ; 2.37-2.31$ (m, $2 \mathrm{H}$ ); 2.25 (“t”, $1 \mathrm{H}, J=8.9 \mathrm{~Hz}$ ); 2.10 (“d”, $1 \mathrm{H}, J=17 \mathrm{~Hz}$ ); $0.92(\mathrm{~d}, 3 \mathrm{H}, J=6.5 \mathrm{~Hz}) ; 0.87-0.85$ (2 doublets, $10 \mathrm{H}) ; 0.54$ $(\mathrm{s}, 3 \mathrm{H})$. Due to the difficulty in separating CTL and HOCTL from their Pro and HOPro precursors, some experiments were performed with 90/10 CTL/Pro mixtures. The presence of $\sim 10 \%$ Pro contamination is readily observed as structure at $\lambda<310 \mathrm{~nm}$ in UV spectra (Fig. S1 in SI) and in ${ }^{1} \mathrm{H}$ NMR spectra.

We observed no differences in irradiations of similar solutions of HOCTL and CTL, indicating that the 25-OH substituent does not influence photochemical and photophysical properties. HORP is the sole photoproduct in carefully $\mathrm{N}_{2}$-outgassed or degassed THF and the endoperoxide, HOCTLP, is the major photoproduct in air-saturated THF, provided that a relatively high concentration of HOCTL is employed $\left(\sim 6 \times 10^{-3} \mathrm{M}\right)$. As in the case of HOCTL, the presence of the $25-\mathrm{OH}$ substituent led to X-ray diffraction quality crystals and enabled definitive product identification. Crystallization of HORP from a 3:1 wet $\mathrm{CH}_{3} \mathrm{CN} /$ benzene mixture afforded small transparent monohydrate crystals. The structure obtained from the X-ray diffraction of these crystals is shown in Fig. 3 and Scheme 1. It differs from the structure proposed by Barton et al. in the stereochemistry 


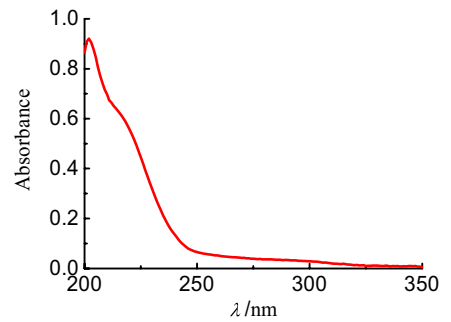

Fig. 4 The UV spectrum of HOCTLP in ethanol

of the bond-crossing reaction. Crystals of HOCTLP were obtained from an ethanol/deionized water mixture X-ray analysis revealed the structure in Fig. 3 in agreement with Fieser's proposal [16]. The UV spectrum of HPLC-purified HOCTLP in ethanol, Fig. 4, shows $\lambda_{\max }$ at $202 \mathrm{~nm}$, a shoulder at $215 \mathrm{~nm}$ and very weak featureless absorption in the 250-350 nm region. Previously reported longer wavelength bands for CTLP, DHEP and the peroxides of their provitamin D precursors are not likely to be correct [19]. Ashour et al. gave $\lambda_{\max }$ at $204 \mathrm{~nm}$, for ProP in methanol [25], but their $243 \mathrm{~nm}$ peak is not supported by our spectrum. The spectrum for DHEP by Albro et al. of end absorption with a shoulder at $222 \mathrm{~nm}$ [26] is closer to our own.

\subsection{HOCTL triplet}

A $3 \mathrm{~mL}$ aliquot of a THF solution containing $5.0 \times 10^{-3} \mathrm{M}$ HOCTL and $2.5 \times 10^{-3} \mathrm{M}$ benzophenone was transferred into a Pyrex tube equipped with a standard taper joint, provided with a grease trap and attached via a graded seal to a standard $1.0 \mathrm{~cm}^{2} \mathrm{UV}$ cell. The solution was degassed using five freeze-pump-thaw cycles to $<10^{-4}$ Torr and flame sealed at a constriction. Pulse excitation at $370 \mathrm{~nm}$ led to transient spectra recorded periodically in the $0-100 \mu$ s time scale, Fig. S5 in SI. The bleach observed at 0 -time is probably due to stimulated fluorescence from ${ }^{1} \mathrm{HOCTL} *$ formed by direct excitation due to the high [HOCTL] employed. At longer times, we observed the known transient absorptions of the benzophenone triplet and the benzophenone ketyl radical [27, 28], and a new transient absorption, assigned to ${ }^{3} \mathrm{HOCTL}^{*}$, with $\lambda_{\max }=395 \mathrm{~nm}$ and a shoulder at $416 \mathrm{~nm}$, Fig. 5a. Decay monitored at $395 \mathrm{~nm}$, Fig. 5b, is monoexponential and gives $\tau=80 \mu \mathrm{s}$. The small peak at $452 \mathrm{~nm}$ does not belong to this transient as it does not appear when HOCTL is excited directly or when benzophenone triplet energy transfer to HOCTL is in ethanol [21].

\subsection{Photoproduct quantum yields}

The quantum yield of RP formation was measured using HOCTL contaminated with $11 \%$ Pro based on ${ }^{1} \mathrm{H}$ NMR and UV analysis. Three $\mathrm{mL}$ aliquots of degassed HOCTL
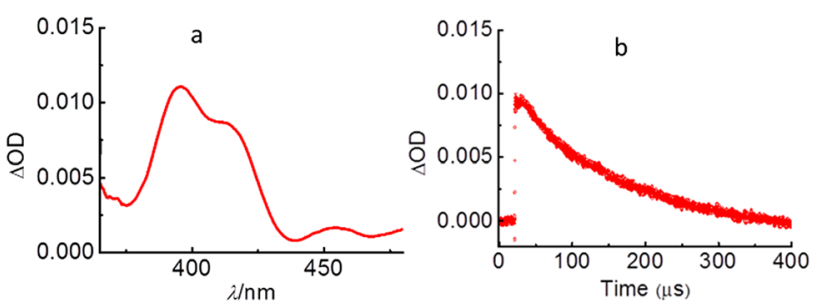

Fig. 5 a The ${ }^{3}$ HOCTL* transient. b Triplet decay at $395 \mathrm{~nm}$

solutions, $3.92 \times 10^{-3} \mathrm{M}$ in THF were irradiated in parallel in the Moses merry-go-round [23] together with a $3.0 \mathrm{~mL}$ $4.27 \times 10^{-2} \mathrm{M}$ degassed pentane solution of trans-stilbene ( $0.011 \%$ cis-stilbene by GC), used for actinometry. The samples were immersed in a water bath whose $T$ was maintained at $25^{\circ} \mathrm{C}$. A $450-\mathrm{W}$ Hanovia lamp was employed together with the $313 \mathrm{~nm}$ filter solution. Pro is transparent at $\lambda_{\text {exc }}=313 \mathrm{~nm}$ and does not interfere in these measurements. Following $12 \mathrm{~h}$ irradiation, HOCTL conversion to HORP (8.3\% - see the Supporting Information) was measured by ${ }^{1} \mathrm{H}$ NMR and trans- $\rightarrow$ cis-stilbene conversion $(11.6 \pm 0.2 \%)$ was measured by GC. Correction for back reaction as previously described [22] gave $12.4 \pm 0.2 \%$ cisstilbene. With the use of $\phi_{\mathrm{tc}}=0.52$ in the actinometer [22], we obtain $\phi_{\mathrm{RP}}=0.025_{2}$. An independent experiment carried out as above but using pure $[\mathrm{HOCTL}]=1.90 \times 10^{-3} \mathrm{M}$, $[t$-St $]=4.01 \times 10^{-2} \mathrm{M}$ and the $550 \mathrm{~W}$ Hanovia lamp gave $9.3 \pm 0.2$ and $8.8_{8} \%$ conversions for RP and $c$-St (corrected for back reaction), respectively, yielding $\phi_{\mathrm{RP}}=0.025_{7}$.

Initial observations revealed that, in the presence of air, the relative yield of peroxide formation increases at higher CTL or HOCTL concentrations. Accordingly, quantum yields of HOCTLP formation, $\phi_{\text {Per }}$, were obtained at three [HOCTL] using HOCTL contaminated with $10 \%$ HOPro in air-saturated THF solutions. Three $\mathrm{mL}$ aliquots were irradiated at $313 \mathrm{~nm}$ in the merry-go-round in parallel with degassed trans-stilbene actinometry solutions, $0.026 \mathrm{M}$ in pentane. The highest HOCTL concentration was irradiated twice as long. Stilbene conversions, corrected for back reaction, were $6.9 \pm 0.3$ and $12.9 \pm 0.7 \%$ for 2 and $4 \mathrm{~h}$ irradiation, respectively. Conversions to peroxide were determined by ${ }^{1} \mathrm{H}$ NMR using the areas of vinyl protons ( $\delta 5.7$ for HOCTL and 6.31 and 6.62 for HOCTLP). The quantum yields are shown in Table 2 . The [HOCTL] values in Table 2 are based on the absorbance of the solutions at $324 \mathrm{~nm}$ using $\varepsilon_{324}=1.1_{0} \times 10^{4} \mathrm{M}^{-1} \mathrm{~cm}^{-1}$. The first row in Table 2 gives results from a separate analogous experiment in which air- and $\mathrm{O}_{2}$-saturated THF solutions of pure HOCTL were irradiated in parallel with the trans-stilbene actinometer. 
Table 2 Peroxide (HOCTLP) quantum yields

\begin{tabular}{llll}
\hline$[\mathrm{HOCTL}] / \mathrm{mM}$ & $\phi_{\text {Per }}$ Air & $\phi_{\text {Per }} \mathrm{O}_{2}$ & $\tau_{\Delta} / \mu \mathrm{s}$ (see text) \\
\hline 1.12 & $0.059_{4}$ & $0.080_{5}$ & 30 \\
$1.31^{\mathrm{a}}$ & 0.058 & & $28 . ._{4}$ \\
$1.96^{\mathrm{a}}$ & 0.083 & & 27.6 \\
$3.92^{\mathrm{a}}$ & 0.110 & & 25.6 \\
\hline
\end{tabular}

${ }^{a}$ Solutions are contaminated by $11 \%$ HOPro- - see text

\section{Discussion}

Windaus and Linsert isolated photodehydroergosterol (RP) in $40 \%$ yield from the UV irradiation of DHE [13]. Their UV spectrum agrees with our spectrum in Fig. $2 \mathrm{~b}$ except that our minimum at $220 \mathrm{~nm}$ is less deep. Conjugation of the diene moiety with a cyclopropane ring was inferred from this UV spectrum by Barton and Kende who used chemical reactions and IR spectra to eliminate two other possible structures in assigning the structure to RP, a remarkable pre- ${ }^{1} \mathrm{H}$ NMR achievement. Our X-ray structure in Fig. 3 confirms the proposed cross-addition of the $\mathrm{C}_{9} \mathrm{C}_{10} \sigma$-bond to the $\mathrm{C}_{5} \mathrm{C}_{6} \pi$-bond and provides correct orientational detail. The product evolution in the UV spectra in THF in Fig. 2a is significantly different from that reported by Morton et al. in ethanol [20]. The shift of the product $\lambda_{\max }$ from $243 \mathrm{~nm}$ in ethanol to 261 in THF reflects mainly the formation of addition and reduction photoproducts in ethanol that are absent in THF [21]. The presence of an isosbestic point in Fig. 2a shows that the photoproduct composition does not change in the course of the reaction. GC (used for CTL only) and ${ }^{1} \mathrm{H}$ NMR analyses were consistent with formation of RP as the sole product in degassed THF solution. In Fig. 2a, quantitative conversion of HOCTL to HORP predicts $\varepsilon_{261}=7.8_{7} \times 10^{3} \mathrm{M}^{-1} \mathrm{~cm}^{-1}$ for HORP. Our independently measured value for HORP in THF, $\varepsilon_{\text {max }}=7.8_{5} \times 10^{3} \mathrm{M}^{-1} \mathrm{~cm}^{-1}$ (Beer-Lambert plots for HOCTL and HORP in THF are given in Figs. S2 and S3, respectively, in the Supporting Information) is in excellent agreement with this value. A much higher value of $\varepsilon_{\max }=1.13 \times 10^{4} \mathrm{M}^{-1} \mathrm{~cm}^{-1}$ was reported by Barton and Kende for the acetate of RP from ergosterol at $\lambda_{\max }=260 \mathrm{~nm}$ in ethanol [15].

\subsection{Mechanism}

Equations (1, 2, 3, 4, 5, 6) account for the photochemistry and photophysics of CTL and HOCTL in degassed THF.

$$
\begin{aligned}
& { }^{1} \mathrm{CTL}^{h \nu} \longrightarrow{ }^{1} \mathrm{CTL}^{*} \\
& { }^{1} \mathrm{CTL}^{*} \stackrel{k_{f}}{\longrightarrow}{ }^{1} \mathrm{CTL}+h v \\
& { }^{1} \mathrm{CTL}^{*} \stackrel{k_{\mathrm{RP}}}{\longrightarrow}{ }^{1} \mathrm{RP}
\end{aligned}
$$

${ }^{1} \mathrm{CTL}^{*} \stackrel{k_{\mathrm{is}}}{\longrightarrow}{ }^{3} \mathrm{CTL}^{*}$

${ }^{1} \mathrm{CTL}^{*} \stackrel{k_{\mathrm{ds}}}{\longrightarrow}{ }^{1} \mathrm{CTL}$

${ }^{3} \mathrm{CTL}^{*} \stackrel{k_{\mathrm{dt}}}{\longrightarrow}{ }^{1} \mathrm{CTL}$.

In the presence of ${ }^{3} \mathrm{O}_{2}$, Eqs. $(7,8,9,10)$ augment this sequence.

${ }^{1} \mathrm{CTL}^{*}+{ }^{3} \mathrm{O}_{2} \stackrel{k_{\mathrm{ox}}^{s}}{\longrightarrow}{ }^{3} \mathrm{CTL}^{*}+{ }^{1} \mathrm{O}_{2}^{*}$

${ }^{3} \mathrm{CTL}^{*}+{ }^{3} \mathrm{O}_{2} \stackrel{k_{o x}^{t}}{\longrightarrow}{ }^{1} \mathrm{CTL}+{ }^{1} \mathrm{O}_{2}^{*}$

${ }^{1} \mathrm{CTL}+{ }^{1} \mathrm{O}_{2}^{*} \stackrel{k_{\mathrm{per}}}{\longrightarrow} \mathrm{CTLP}$

${ }^{1} \mathrm{O}_{2}^{*} \stackrel{k^{\mathrm{dox}}}{\longrightarrow}{ }^{3} \mathrm{O}_{2}$

In Eq. (7) quenching of the triplet state is shown, tentatively, with concomitant formation of ${ }^{1} \mathrm{O}_{2}{ }^{*}$. This is possible [29] when the $S_{1}-T_{1}$ energy gap exceeds $22.6 \mathrm{kcal} / \mathrm{mol}$, the energy of ${ }^{1} \Delta_{\mathrm{g}}{ }^{1} \mathrm{O}_{2} *$ [30], a condition that is satisfied in a conjugated triene moiety. Based on the overlap of absorption and fluorescence spectra of CTL, the energy of the $S_{1}$ state is about $82 \mathrm{kcal} / \mathrm{mol}$. The energy of $\mathrm{T}_{1}$ probably lies $40 \mathrm{kcal} /$ mol lower, because triplet sensitized cis-trans photoisomerization of the 1,3,5-hexatrienes [31] and of previtamin and tachysterol $\mathrm{D}_{3}$ [32] were achieved with sensitizers having $E_{\mathrm{T}} \mathrm{s}$ as low as $42 \mathrm{kcal} / \mathrm{mol}$.

\subsection{Photophysics}

On the basis of the above mechanism, the effect of $\mathrm{O}_{2}$ on HOCTL fluorescence quantum yields is given by Eq. (11), the Stern-Volmer equation

$\frac{\phi_{f}^{0}}{\phi_{f}}=1+k_{\mathrm{ox}}^{S} \tau_{\mathrm{f}}^{0}\left[\mathrm{O}_{2}\right]$,

where $\phi_{\mathrm{f}}{ }^{0}=k_{\mathrm{f}} \tau_{\mathrm{f}}^{0}$ and $\tau_{\mathrm{f}}^{0}=\left(k_{\mathrm{f}}+k_{\mathrm{RP}}+k_{\mathrm{is}}+k_{\mathrm{d}}\right)^{-1}$ are the fluorescence quantum yield and lifetime in the absence of $\mathrm{O}_{2}$. The analogous expression for the effect of $\mathrm{O}_{2}$ on the fluorescence lifetime is given in Eq. (12).

$\frac{1}{\tau_{\mathrm{f}}}=\frac{1}{\tau_{\mathrm{f}}^{0}}+k_{\mathrm{ox}}^{S}\left[\mathrm{O}_{2}\right]$,

We calculated the $\left[\mathrm{O}_{2}\right]$ values in Table 1 for air- and $\mathrm{O}_{2}$-saturated THF using $\mathrm{O}_{2}$ mole fraction data in THF [33] and THF densities [34] and vapour pressures at $20^{\circ} \mathrm{C}$ $[34,35]$. Plots of the data in Table 1 according to Eqs. (11 

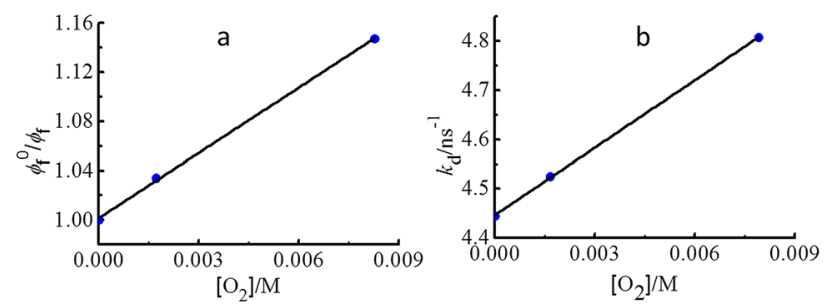

Fig. 6 Plots of the data in Table 1: a Stern-Volmer plot and b decay rates

and 12) are shown in Fig. 6. The slope of the lifetime plot in Fig. $6 \mathrm{~b}$ gives $k_{\mathrm{ox}}^{S}=4.5 \times 10^{10} \mathrm{M}^{-1} \mathrm{~s}^{-1}$ and the plot in Fig. $6 \mathrm{a}$ gives $7.9 \times 10^{10} \mathrm{M}^{-1} \mathrm{~s}^{-1}$. The average of these two values, $k_{\mathrm{ox}}^{S}=6.2 \times 10^{10} \mathrm{M}^{-1} \mathrm{~s}^{-1}$, is about twice as large as rate constants for $\mathrm{O}_{2}$ quenching of excited singlet states of arenes in cyclohexane [29]. The higher value in THF is due almost entirely to its lower viscosity [36, 37] (the cyclohexane to THF viscosity ratio is 1.91 at $20^{\circ} \mathrm{C}$ ) [34]. We conclude that the quenching of ${ }^{1} \mathrm{HOCTL}^{*}$ by $\mathrm{O}_{2}$ is at or close to the dif-
$[41,42]$. These workers did not provide the experimental $\phi_{\mathrm{f}}$ and $\tau_{\mathrm{f}}$ values in ethanol, but their anomalously low $\varepsilon_{\max }=7.7 \times 10^{3} \mathrm{M}^{-1} \mathrm{~cm}^{-1}$ for CTL in Fig. 1 should have led to a low rather than a high calculated $k_{\mathrm{f}}$ value.

\subsection{Photochemistry}

Application of the steady-state approximation on all excited species in the above mechanism leads to the quantum yield expression in Eq. 13 for RP formation. Our quantum yields

$\phi_{\mathrm{RP}}=\frac{k_{\mathrm{RP}} \tau_{\mathrm{f}}}{1+k_{\mathrm{ox}}^{s} \tau_{\mathrm{f}}\left[\mathrm{O}_{2}\right]}$,

for RP formation in degassed THF, $0.025_{7}$, and $\tau_{\mathrm{s}}=0.22_{5} \mathrm{~ns}$ from Table 1 give $k_{\mathrm{RP}}=1.1_{4} \times 10^{8} \mathrm{~s}^{-1}$ for the HOCTL rearrangement rate constant.

The quantum yield of peroxide formation is a function of the effective intersystem crossing quantum yield, the sum of the fractions of ${ }^{1} \mathrm{CTL}^{*}$ and ${ }^{3} \mathrm{CTL} *$ that give ${ }^{1} \mathrm{O}_{2} *$ by energy transfer, and the fraction of ${ }^{1} \mathrm{O}_{2} *$ that reacts with ${ }^{1} \mathrm{CTL}$ to give CTLP, Eq. 14

$\phi_{\mathrm{CTLP}}=\left(\frac{k_{\mathrm{per}} \tau_{\Delta}[\mathrm{CTL}]}{1+k_{\mathrm{per}} \tau_{\Delta}[\mathrm{CTL}]}\right)\left\{\frac{k_{\mathrm{ox}}^{S} \tau_{\mathrm{s}}\left[\mathrm{O}_{2}\right]}{1+k_{\mathrm{ox}}^{S} \tau_{\mathrm{s}}\left[\mathrm{O}_{2}\right]}+\frac{k_{\mathrm{ox}}^{T} \tau_{\mathrm{t}}\left[\mathrm{O}_{2}\right]}{1+k_{\mathrm{ox}}^{T} \tau_{\mathrm{t}}\left[\mathrm{O}_{2}\right]}\left(\frac{k_{\mathrm{is}} \tau_{\mathrm{s}}+k_{\mathrm{ox}}^{S} \tau_{\mathrm{s}}\left[\mathrm{O}_{2}\right]}{1+k_{\mathrm{ox}}^{S} \tau_{\mathrm{s}}\left[\mathrm{O}_{2}\right]}\right)\right\}$,

fusion controlled limit [29]. A $4.5 \times 10^{10} \mathrm{M}^{-1} \mathrm{~s}^{-1} k_{\mathrm{ox}}^{S}$ value in THF at $25{ }^{\circ} \mathrm{C}$ was reported recently for $\mathrm{O}_{2}$ quenching of cycloparaphenylene fluorescence [38]. Correcting the $\left[\mathrm{O}_{2}\right]$ values in ref 38 by including the THF vapour pressure raises that value to $5.7 \times 10^{10} \mathrm{M}^{-1} \mathrm{~s}^{-1}$ improving the agreement with our value.

The $\phi_{\mathrm{f}} / \tau_{\mathrm{f}}$ ratios in Table 1 give $k_{\mathrm{f}}=1.4_{5} \times 10^{8} \mathrm{~s}^{-1}$. Our results can be compared with fluorescence quantum yields and lifetimes measured for DHE in several air-saturated solvents [39]. Not surprisingly, in view of the identical triene chromophores in the two molecules, they were found to be the same as those for CTL in three solvents used for comparison [39]. Discounting anomalously low DHE $\phi_{\mathrm{f}}$ values in acetone and chloroform, $\phi_{\mathrm{f}}$ values in the remaining seven solvents fall in the $0.024-0.058$ range and the range of corresponding $\tau_{\mathrm{f}}$ values is $0.18-0.39 \mathrm{~ns}$. The uncertainty in these values is evident when one considers that the range of $k_{\mathrm{f}}$ values calculated from the $\phi_{\mathrm{f}} / \tau_{\mathrm{f}}$ ratios, $1.3-2.2 \times 10^{8} \mathrm{~s}^{-1}$, is much wider than expected based on the nearly solvent independent $\varepsilon_{\max }=1.09 \pm 0.06 \times 10^{4} \mathrm{M}^{-1} \mathrm{~cm}^{-1}$. [38] Our $\phi_{\mathrm{f}}$ and $\tau_{\mathrm{f}}$ values in THF are very close to the values for diethyl ether reported by Smutzer et al. [39]. Hyslop et al. reported $k_{\mathrm{f}}=1.79 \times 10^{8} \mathrm{~s}^{-1} \mathrm{CTL}$ in ethanol at $37^{\circ} \mathrm{C}$, [40] a value that exceeds ours by $\sim 23 \%$. Almost the same value was calculated [40] using the Strickler-Berg relationship where the $\tau$ 's, in order of appearance, are the lifetimes of ${ }^{1} \mathrm{O}_{2}{ }^{*}$ and of the singlet and triplet excited states of CTL. The second term in Eq. (14), $\phi_{\Delta}$, is the quantum yield of ${ }^{1} \Delta_{\mathrm{g}}$ ${ }^{1} \mathrm{O}_{2}$ * formation and is a constant at constant $\left[\mathrm{O}_{2}\right]$. To evaluate the effect of HOCTL concentration on peroxide quantum yields, Eq. (14) can be rearranged to Eq. (15).

The quantum yields obtained in the presence of air in Table 2 are plotted according to Eq. (15) in Fig. 7a.

$\frac{1}{\phi_{\text {HOCTLP }}}=\left(1+\frac{1}{k_{\text {per }} \tau_{\Delta}[\text { HOCTL }]}\right) \frac{1}{\phi_{\Delta}} ;$

The intercept/slope ratio of the linear plot gives $k_{\mathrm{per}} \tau_{\Delta}=420 \mathrm{M}^{-1}$. Reported lifetimes of ${ }^{1} \mathrm{O}_{2}{ }^{*}$ are 30 [43] and $20[44] \mu$ s. The experimental rate constant for the
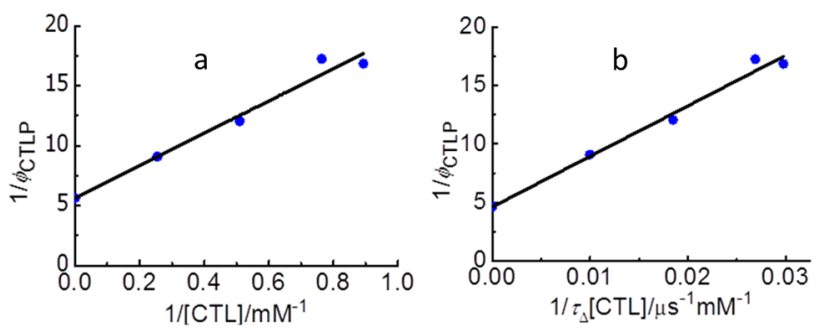

Fig. 7 a The effect of [HOCTL] on $\phi_{\text {CTLP }}$, b as in a but accounting for variation in $\tau_{\Delta}$ 
stereospecific insertion of ${ }^{1} \mathrm{O}_{2} *$ into a $\alpha-\mathrm{H}$ of THF [45] is $3.8 \times 10^{3} \mathrm{M}^{-1} \mathrm{~s}^{-1}$. That reaction gives the hydroperoxide and $\gamma$-butyrolactone, its rearrangement product, with a reported quantum yield of 0.93 [45]. If there were no other decay paths, in neat THF, this corresponds to $\tau_{\Delta}=23 \mu \mathrm{s}$, indicating that the insertion reaction accounts for most, if not all, the lifetime of ${ }^{1} \mathrm{O}_{2}{ }^{*}$. With the use of $\tau_{\Delta}=30 \mu$ s, we obtain $k_{\text {per }}=1.4 \times 10^{7} \mathrm{M}^{-1} \mathrm{~s}^{-1}$. However, in contrast to the entries in the first row of Table 2 that were obtained with pure HOCTL, entries for the other three rows were obtained with HOCTL samples that were contaminated by $11 \%$ HOPro. While the presence of HOPro in the experiments for Fig. 7 does not interfere with incident light absorption, its reaction with ${ }^{1} \mathrm{O}_{2}{ }^{*}$ alters the effective lifetime of ${ }^{1} \mathrm{O}_{2}{ }^{*}$. Reported rate constants for the reactions of ergosterol with ${ }^{1} \mathrm{O}_{2}{ }^{*}$ are $1.2 \times 10^{7} \mathrm{M}^{-1} \mathrm{~s}^{-1}$ and $2.1 \times 10^{7} \mathrm{M}^{-1} \mathrm{~s}^{-1}$ in tert-butyl methyl ether [26] and benzene [46], respectively. Assuming that the reactivities of Pro and HOPro with ${ }^{1} \mathrm{O}_{2}{ }^{*}$ are the same as the reactivity of ergosterol, the ${ }^{1} \mathrm{O}_{2}{ }^{*}$ lifetime in Eq. (15) is not a constant, but varies with the HO-Pro concentration, Eq. (16).

$\tau_{\Delta}=\frac{1}{k_{\mathrm{dox}}+k_{\mathrm{Pro}}[\mathrm{HOPro}]}$

With the use of $\tau_{\Delta}=30 \mu$ s in the absence of HOPro impurity [43], and the rate constant in tert-butyl methyl ether, lifetimes based on Eq. (16) are given in the last column of Table 2. The intercept/slope ratio of the plot of the inverse of the peroxide quantum yield against $1 /\left(\tau_{\Delta}\right.$ [HOCTL] $)$ in Fig. $7 \mathrm{~b}$ gives $k_{\text {per }}=1.0_{7} \times 10^{7} \mathrm{M}^{-1} \mathrm{~s}^{-1}$. This is a bit larger than expected in view of an earlier finding by Albro et al. that in tert-butyl methyl ether, the reaction of DHE with ${ }^{1} \mathrm{O}_{2} *$ is half as fast as the reaction with ergosterol [26]. Albro et al. also found that the conjugated trienols, present as minor impurities in commercial ergosterol and provitamin $\mathrm{D}_{3}$ samples, function as photosensitizers for the ${ }^{1} \mathrm{O}_{2}$ * oxidation of ergosterol and provitamin $\mathrm{D}_{3}$ in vitro, and, because they are present in the skin, in vivo. [26] The conclusion that endoperoxide formation is faster with HOPro than with HOCTL is not surprising, because angle strain due to the exocyclic double bond in HOCTLP should raise its energy, thereby raising the energy of the transition state for its formation.

The first row of Table 2 shows a significant increase in $\phi_{\text {HOCTLP }}$ when the reaction is carried out under an $\mathrm{O}_{2}$ atmosphere. Our ${ }^{3}$ HOCTL* lifetime from Fig. $5 \mathrm{~b}$ and the expectation [29] that $k_{\mathrm{ox}}{ }^{t}=(1 / 9) k_{\mathrm{ox}}{ }^{\mathrm{s}}$ lead to the conclusion that, in air-saturated THF solutions, all ${ }^{3} \mathrm{HOCTL} *$ are quenched by oxygen. We further simplify Eq. (14) by noting that the first term, $f_{\text {CTL }}$, is constant at constant [HOCTL]. We then factor out $f_{\mathrm{O} 2}=k_{\mathrm{ox}}{ }^{\mathrm{s}} \tau_{\mathrm{s}}\left[\mathrm{O}_{2}\right] /\left(1+k_{\mathrm{ox}}{ }^{\mathrm{s}} \tau_{\mathrm{s}}\left[\mathrm{O}_{2}\right]\right)$ and cross multiply by the $f$ terms to obtain Eq. (17)

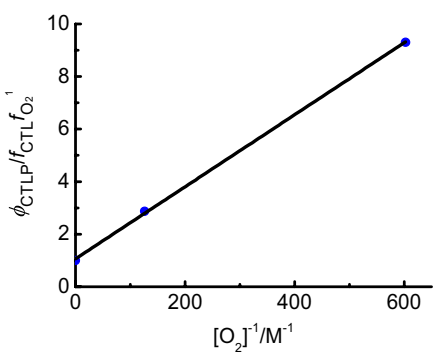

Fig. 8 The effect of oxygen concentration on $\phi_{\mathrm{CTLP}}$

$\frac{\phi_{\text {HОстLP }}}{f_{\text {НОстL }} f_{\mathrm{O} 2}}=\left(n+\frac{k_{\text {is }}}{k_{\mathrm{OX}}^{s}\left[\mathrm{O}_{2}\right]}\right)$,

where $n$ is 2 if oxygen quenching of ${ }^{1}$ HOCTL $^{*}$ gives ${ }^{3}$ HOCTL*, as shown in Eq. (7), but $n$ is 1 if it gives groundstate HOCTL. Plotted according to Eq. (17) in Fig. 8, the quantum yields in Table 2 are well fit with an intercept of 1. We conclude that Eq. (7) is not correct. Quenching of ${ }^{1} \mathrm{HOCTL}^{*}$ by oxygen does not give ${ }^{3} \mathrm{HOCTL}^{*}$ as we had expected. The slope of the line in Fig. 8 gives $\left(k_{\mathrm{is}} / k_{\mathrm{ox}}{ }^{\mathrm{s}}\right)=0.0139 \mathrm{M}$ from which we obtain $k_{\mathrm{is}}=8.9 \times 10^{8} \mathrm{~s}^{-1}$ and using the lifetime in Table $1, \phi_{\text {is }}=0.20$. This value is subject to $\pm 25 \%$ error, because it is affected by the large uncertainty in our $k_{\mathrm{ox}}^{S}$. It is in good agreement with 0.22 , the quantum yield for ${ }^{1} \mathrm{O}_{2} *$ formation that we obtain from the inverse of the intercept in Fig. 7b. A significantly smaller quantum yield of 0.09 for the formation of ${ }^{1} \mathrm{O}_{2} *$ using dehydroergosterol as the photosensitizer in tert-butyl methyl ether was based on the relative phosphorescence intensity of ${ }^{1} \mathrm{O}_{2} *[26]$.

\section{Conclusion}

Photophysical and photochemical quantities determined in this work for HOCTL in THF are summarized in Table 3. We expect them to apply also to CTL and DHE. As conjugated trienes, these steroidal trienols are unusual in that they fluoresce and intersystem cross in solution. By comparison, their diene precursors, Pro and ergosterol, fluoresce only in low $T$ glassy media [47-50] and undergo no intersystem crossing [46]. The unusual behavior is no doubt due to the structural rigidity that inhibits the torsional relaxation pathways that dominate the photochemistry and radiationless decay of the parent 1,3.5-hexatrienes [51]. It is reminiscent of the dramatic enhancement of fluorescence that structural rigidity induces on the trans-stilbene chromophore [52]. Structural rigidity also increases almost 1000 -fold the lifetime of the 
Table 3 Quantum yields and rate constants: HOCTL in THF

\begin{tabular}{llll}
\hline Quantum yield & \multicolumn{3}{c}{ Rate constant } \\
\hline$\phi_{\mathrm{f}}$ & $0.033_{5}$ & $k_{\mathrm{f}}$ & $1.4_{5} \times 10^{8} \mathrm{~s}^{-1}$ \\
$\phi_{\text {is }}$ & 0.20 & $k_{\text {is }}$ & $8.9 \times 10^{8} \mathrm{~s}^{-1}$ \\
$\phi_{\mathrm{RP}}$ & $0.025_{7}$ & $k_{\mathrm{RP}}$ & $1.1_{4} \times 10^{8} \mathrm{~s}^{-1}$ \\
$\phi_{\text {ic }}$ & 0.74 & $k_{\text {ic }}$ & $3.3 \times 10^{9} \mathrm{~s}^{-1}$ \\
& & $k_{\mathrm{dt}}$ & $1.25 \times 10^{4} \mathrm{~s}^{-1}$ \\
\hline
\end{tabular}

trienol triplet state [53] rendering it a more effective ${ }^{1} \mathrm{O}_{2}{ }^{*}$ sensitizer. As noted above, the strong structural similarity of the steroidal trienols to cholesterol has made them ideal fluorescent probes for cholesterol trafficking in vivo. Although weakly fluorescent in solution, they fluoresce more brightly in the confining environments of biological membranes and phospholipid bilayers, generally. Fluorescence lifetimes of 0.97 [39] and 0.9 [54] ns, measured at room temperature in such media, and the radiative rate constant in Table 3 allow estimation of $\phi_{\mathrm{f}}=0.13-0.14$ at room temperature, substantially higher than the solution value in Table 3. Similarly estimated, but based on $k_{\mathrm{f}}=1.78 \times 10^{8} \mathrm{~s}^{-1}$, is $\phi_{\mathrm{f}}=0.11$ for CTL in liposomes at $37{ }^{\circ} \mathrm{C}$ [40]. That value drops to $\phi_{\mathrm{f}}=0.090$ with the use of our $k_{\mathrm{f}}$. By analogy with ergosterol whose low $T$ fluorescence competes with activated radiationless decay [48], the decrease in $\phi_{\mathrm{f}}$ at the higher temperature is expected. Relevant to the use of the cholesteryl trienols as fluorescent probes for cholesterol trafficking is the report that the CTL fluorescence lifetime increases to $2.2 \mathrm{~ns}$ for CTL in cholesterol aggregates at $37{ }^{\circ} \mathrm{C}$ [40]. Use of our $k_{\mathrm{f}}$ value gives $\phi_{\mathrm{f}}=0.32$ under those conditions.

The lifetime increases must come at the expense of the sum of the three radiationless processes listed in Table 3. Of these, the major one, internal conversion to the ground state, must surely be diminished in more rigid media, but whether formation of the rearrangement product is similarly affected remains to be established. It is also possible that internal conversion and rearrangement are simultaneous processes that reflect the partitioning between product and reactant on decay through a single conical intersection.

The important role of intersystem crossing in solution was known before our work, and is further documented here in THF. Although the tenfold increase in the fluorescence lifetime in cholesterol aggregates [40] leads us to expect a higher $\phi_{\text {is }}$ under those conditions, the value of $k_{\text {is }}$ must be considerably lower than in THF as $\phi_{\text {is }}$ cannot exceed unity. As observed by Albro et al. [26] the steroidal trienol triplets are efficient sensitizers of ${ }^{1} \mathrm{O}_{2}{ }^{*}$ which, in turn, gives endoperoxides by 1,4 -addition to the cyclohexadiene moiety of the $\mathrm{B}$ ring of the trienols and of the provitamin Ds. The presence of CTL as an impurity in commercial Pro probably accounts for the formation of Pro endoperoxide on $254 \mathrm{~nm}$ irradiation of a Pro solution in $\mathrm{CDCl}_{3}$ [55]. DHE photobleaching observed by Hao and coworkers when DHE was used as a fluorescent probe to monitor cholesterol trafficking in living cells [12] showed that it is photochemically active under physiological conditions. The relative role of rearrangement and peroxide formation in biological media is unknown.

Side photoproducts formed on prolonged irradiation of the vitamin D precursors have been collectively referred to as toxisterols [56-58], although we are aware of no definitive studies confirming their toxicity. Thus, the biological properties of the RP photoproducts are unknown. On the other hand, isolated as natural products, the peroxide photoproducts have been shown to be moderately cytotoxic against a variety of cancer cells $[19,25,55,59]$. It has also been speculated that endoperoxide formation in exposed skin may account for the tumor promoting action of UVA $(\lambda>315 \mathrm{~nm})$ [26].

Supplementary Information The online version contains supplementary material available at https://doi.org/10.1007/s43630-021-00131-w.

Acknowledgements The National Science Foundation, most recently by Grant No. CHE-1361962, and the Florida State University supported this research. This research benefitted from the use of resources provided by the NMR Facility, the X-Ray Crystallography Center, the Mass Spectrometry Laboratory, and the Materials Characterization Laboratory at the FSU Department of Chemistry and Biochemistry. We thank Professor Edwin F. Hilinski for helpful discussions.

Author contributions SBK performed most experimental measurements described in this paper. Initial experiments were performed by SG and EAH. RJC carried out the X-ray crystallographic measurements and determined the X-ray structures. JS directed the research and prepared the manuscript for publication.

\section{Declarations}

Conflict of interest The authors declare no competing financial interests.

\section{References}

1. Ruan, B., Wilson, W. K., Pang, J., Gerst, N., Pinkerton, F. D., Tsai, J., Kelley, R. I., Whitby, F. G., Milewicz, D. M., Garbern, J., \& Schroepfer, G. J. (2001). Sterols in blood of normal and SmithLemli-Opitz subjects. Journal of Lipid Research, 42, 799-812.

2. Ruan, B., Wilson, W. K., Tsai, J., \& Schroepfer, G. J. (2000). Aberrant pathways in the late stages of cholesterol biosynthesis. Origin and metabolic fate of unsaturated sterols relevant to the Smith-Lemli-Opitz syndrome. Journal of Lipid Research, 41, 1772-1782.

3. Delseth, C., Kashman, Y., \& Djerassi, C. (1979). Ergosta5,7,9(11),22-tetraen-3 $\beta$-ol and its 24 -ethyl homolog. Two new marine sterols from the red sea sponge Biemna fortis. Helvetica Chimica Acta, 62, 2037-2045.

4. Heald, S. L., Jeffs, P. W., \& Wheat, R. W. (1981). The identification of ergosterol and $\Delta^{9(11)}$-dehydroergosterol from mycelia of Coccidioides immitis by reverse-phase high-performance liquid 
and gas chromatography and ultraviolet and mass spectrometry. Experimental Mycology, 5, 162-166.

5. Gunatilaka, A. A. L., Gopichand, Y., Schmitz, F. J., \& Djerassi, C. (1981). Minor and Trace sterols in marine invertebrates. Isolation and structure elucidation of nine new $5 \alpha, 8 \alpha$-epidioxy sterols from four marine organisms. Journal of Organic Chemistry, 46, 3860-3866.

6. Sica, D., Boniforti, L., \& DiGiacomo, G. (1982). Sterols of Candida tropicalis grown on $n$-alkanes. Phytochemistry, 21, 234-236.

7. Fischer, R. T., Stephenson, F. A., Shafiee, A., \& Schroeder, F. (1984). $\Delta^{5,7,9(11)}$-Cholestatrien-3- $\beta$-ol: A fluorescent cholesterol analogue. Chemistry and Physics of Lipids, 36, 1-14.

8. Fischer, R. T., Cowlen, M. S., Dempsey, M. E., \& Schroeder, F. (1985). Fluorescence of $\Delta^{5,7,9(11), 22}$-ergostatetraen-3 $\beta$-ol in micelles, complexes, and plasma membranes. Biochemistry, 24, 3322-3331.

9. Schroeder, F. (1984). Fluorescent sterols-probe molecules of membrane-structure and function. Progress in Lipid Research, $23,97-113$.

10. Wüstner, D. (2007). Fluorescent sterols as tools in membrane biophysics and cell biology. Chemistry and Physics of Lipids, 146, $1-25$.

11. Maxfield, F. R., \& Wüstner, D. (2012). Analysis of cholesterol trafficking with fluorescent probes. Methods in Cell Biology, 108, 367-393.

12. Hao, M., Lin, S. X., Karylowski, O. J., Wüstner, D., McGraw, T. E., \& Maxfield, F. R. (2002). Vesicular and non-vesicular sterol transport in living cells. The endocytic recycling compartment is a major sterol organelle. Journal of Biological Chemistry, 277, 609-617.

13. Windaus, A., \& Linsert, O. (1928). Ultraviolet irradiation of dehydroergosterol. Justus Liebigs Annalen der Chemie., 465, 148-166.

14. Windaus, A., Gaede, J., Köser, J., \& Stein, G. (1930). Crystallized irradiation products from ergosterol and dehydroergosterol. Justus Liebigs Annalen der Chemie., 483, 17-30.

15. Barton, D. H. R., \& Kende, A. S. (1958). Photochemical transformations. Part III. The constitution of a steroidal irradiation product. Journal of the Chemical Society, 688-692.

16. Fieser, L. F. (1950). Steric course of reactions of steroids. Experientia, 6, 312-315.

17. Chignell, C. F., Kukielczak, B. M., Sik, R. H., Bilski, P. J., \& He, Y.-Y. (2006). Ultraviolet A sensitivity in Smith-Lemli-Opitz syndrome: Possible involvement of cholesta-5,7,9(11)-trien-3 $\beta$-ol. Free Radical Biology and Medicine, 41, 339-346.

18. Böcking, T., Barrow, K. D., Netting, A. G., Chilcott, T. C., Coster, H. G. L., \& Höfer, M. (2000). Effects of singlet oxygen on membrane sterols in the yeast Saccharomyces cerevisiae. European Journal of Biochemistry, 267, 1607-1618.

19. Chen, Y.-K., Kuo, Y.-H., Chiang, B.-H., Lo, J. M., \& Sheen, L.-Y. (2009). Cytotoxic activities of 9,11-dehydroergosterol peroxide and ergosterol peroxide from the fermentation mycelia of Ganoderma lucidum cultivated in the medium containing leguminous plants on Hep $3 \beta$ cells. Journal of Agricultural and Food Chemistry, 57, 5713-5719.

20. Morton, R. A., Heilbron, I. M., \& Spring, F. S. (1930). Absorption spectra in relation to vitamin A. The Biochemical Journal, 24, 136-140.

21. Saltiel, J., Krishnan, S. B., Gupta, S., Chakraborty, A., \& Lin, X. Unpublished observations.

22. Saltiel, J., Marinari, A., Chang, D.W.-L., Mitchener, J. C., \& Megarity, E. D. (1979). Trans-cis photoisomerization of the stilbenes and a reexamination of the positional dependence of the heavy-atom effect. Journal of the American Chemical Society, $101,2982-2996$
23. Moses, F. G., Liu, R. S. H., \& Monroe, B. M. (1969). The, "merrygo-round" quantum yield apparatus. Molecular Photochemistry, $1,245-249$.

24. Antonucci, R., Bernstein, S., Giancola, D., \& Sax, K. J. (1951). $\Delta^{5,7}$-steroids. VII. The Conversion of $\Delta^{5,7}$ - to $\Delta^{5,7,9}$-Steroidal Hormones. The Journal of Organic Chemistry, 16, 1159-1164.

25. Ashour, M. A., Elkhayat, E. S., Ebel, R., Edrada, R., \& Proksch, P. (2007). Indole alkaloid from the red sea sponge Hyrtios erectus. ARKIVOC, XV, 225-231.

26. Albro, P. W., Bilski, P., Corbett, J. T., Schroeder, J. L., \& Chignell, C. F. (1997). Photochemical reactions and phototoxicity of sterols: Novel self-perpetuating mechanism for lipid photooxidation. Photochemistry and Photobiology, 66, 316-325.

27. Godfrey, T. S., Hilpern, J. W., \& Porter, G. (1967). Triplettriplet absorption spectra of benzophenone and its derivatives. Chemical Physics Letters, 1, 490-492.

28. Topp, M. (1975). Activation-controlled hydrogen abstraction by benzophenone triplet. Chemical Physics Letters, 32, 144-149.

29. Saltiel, J., \& Atwater, B. W. (1988). Spin-statistical factors on diffusion-controlled reactions. Advances in Photochemistry, 14, $1-90$.

30. Herzberg, G. (1950). Spectra of diatomic molecules. Princeton, N. J: Van Nostrand.

31. Møller, S., Langkilde, F. W., \& Wilbrandt, R. (1991). Sensitized triplet photochemistry of $E$ - and Z-1,3,5-hexatriene. Journal of Photochemistry and Photobiology A, 62, 93-106.

32. Saltiel, J., Cires, L., \& Turek, A. M. (2004). Conformer specific photochemistry in the vitamin D field. In W. M. Horspool \& F. Lenci (Eds.), Handbook of organic photochemistry and photobiology, chapter 27 (2nd ed., pp. 1-22). London: CRC Press.

33. IUPAC Analytical Chemistry Division. (1981). Commission on Solubility data, Oxygen and Ozone. In R. Battino (Ed.), Solubility Data Series, 7th edn. Oxford: Pergamon.

34. Weast, R. C. (Ed.). (2021). CRC handbook of chemistry and physics (57th ed., pp. 1976-1977). CRC Press.

35. Scott, D. W. (1970). Tetrahydrofuran: Vibrational assignment, chemical thermodynamic properties, and vapor pressure. The Journal of Chemical Thermodynamics, 2, 833-837.

36. Carvajal, C., Tölle, K. J., Smid, J., \& Swarc, M. (1965). Studies of solvation phenomena of ions and ion pairs in dimethoxyethane and tetrahydrofuran. Journal of the American Chemical Society, 87, 5548-5553.

37. Metz, D. J., \& Glines, A. (1967). Density, viscosity, and dielectric constant of tetrahydrofuran between -78 and $30{ }^{\circ} \mathrm{C}$. Journal of Physical Chemistry, 71, 1158.

38. Suenobu, T., Arahori, I., Nakayama, K., Suzuki, T., Katoh, R., \& Nakagawa, T. (2020). Reaction of oxygen with the singlet excited state of $[n]$ cycloparaphenylenes $(n=9,12$, and 15$)$ : A time-resolved transient absorption study seamlessly covering time ranges from subnanoseconds to microseconds by the randomly-interleaved-pulse-train method. Journal of Physical Chemistry A, 124, 46-55.

39. Smutzer, G., Crawford, B. F., \& Yeagle, P. L. (1986). Physical properties of the fluorescent sterol probe dehydroergosterol. Biochimica et Biophysica Acta, 862, 361-371.

40. Hyslop, P. A., Morel, B., \& Sauerheber, D. (1990). Organization and interaction of cholesterol and phosphatidylcholine in model bilayer membranes. Biochemistry, 29, 1025-1038.

41. Strickler, S. J., \& Berg, R. A. (1962). Relationship between absorption intensity and fluorescence lifetime of molecules. The Journal of Chemical Physics, 37, 814-822.

42. Birks, J. B., \& Dyson, D. J. (1963). The relations between the fluorescence and absorption properties of organic molecules. Proceedings of the Royal Society of London A, 275, 135-148. 
43. Solokhiddinov, K. I., Byteva, I. M., \& Gurinovich, G. P. (1981). Lifetime of singlet oxygen in various solvents. Zhurnal Prikladnoi Spektroskopii, 34, 892-897.

44. Hurst, J. R., McDonald, J. D., \& Schuster, G. B. (1982). Lifetime of singlet oxygen in solution directly determined by laser spectrosopy. Journal of the American Chemical Society, 104, 2065-2067.

45. Sagadevan, A., Huang, K. C., \& Su, M.-D. (2017). Singlet oxygen-mediated selective $\mathrm{C}-\mathrm{H}$ bond hydroperoxidation of ethereal hydrocarbons. Nature Communications, $8,1-8$.

46. Gorman, A. A., Hamblett, I., \& Rodgers, M. A. J. (1987). Ergosterol (Provitamin $\mathrm{D}_{2}$ ) triplet state: An efficient sensitiser of singlet oxygen, $\mathrm{O}_{2}\left({ }^{1} \Delta_{\mathrm{g}}\right)$ formation. Journal of Photochemistry and Photobiology, 45, 215-221.

47. Havinga, E., de Kock, R. J., \& Rappoldt, M. (1960). The photochemical interconversions of provitamin $\mathrm{D}$, lumisterol, previtamin $\mathrm{D}$ and tachysterol. Tetrahedron, 11, 276-284.

48. Nakashima, N., Meech, S. P., Auty, A. R., Nes, A. C., \& Phillips, D. (1985). Fluorescence properties of ergosterol. Journal of Photochemistry, 30, 215-227.

49. Redwood, C., Bayda, M., \& Saltiel, J. (2013). Photoisomerization of pre- and provitamin $\mathrm{D}_{3}$ in EPA at $77 \mathrm{~K}$ : One bond-twist not hula-twist. Journal of Physical Chemistry Letters, 117, 716-721.

50. Bayda, M., Redwood, C. E., Gupta, S., Dmitrenko, O., \& Saltiel, J. (2017). Lumisterol to tachysterol photoisomerization in EPA glass at $77 \mathrm{~K}$. A comparative study. The Journal of Physical Chemistry A, 121, 2331-2342.

51. Saltiel, J., Redwood, C. E., Laohhasurayotin, K., \& Samudrala, R. (2018). Photochemistry of the 1,6-dideuterio-1,3,5-hexatrienes in solution: Efficient terminal bond photoisomerization in one-bondtwist and bicycle pedal ways. Journal of Physical Chemistry A, $122,8477-8489$.
52. Saltiel, J., Zafiriou, O. C., Megarity, E. D., \& Lamola, A. A. (1968). Tests of the singlet mechanism for cis-trans photoisomerization of the stilbenes. Journal of the American Chemical Society, 90, 4759-4760.

53. Langkilde, F. W., Jensen, N.-H., \& Wilbrandt, R. (1987). Timeresolved resonance Raman spectroscopy of the lowest excited triplet states of the $E$ and $Z$ isomers of 1,3,5-hexatriene. Journal of Physical Chemistry, 91, 1040-1047.

54. Yeagle, P. L., Albert, A. D., Boesze-Battaglia, K., Young, J., \& Frye, J. (1990). Cholesterol dynamics in membranes. Biophysical Journal, 57, 413-424.

55. Matsueda, S., \& Katsukura, Y. (1985). Antitumor-active photochemical oxidation products of provitamin D. Chemistry and Industry, 12, 411 (London).

56. Jacobs, H. J. C., \& Havinga, E. (1979). Photochemistry of vitamin $\mathrm{D}$ and its isomers and of simple trienes. Advances in Photochemistry, 11, 305-373.

57. Dauben, W. G., McInnis, E. L., \& Michno, D. M. (1980). Photochemical rearrangements in trienes. In P. de Mayo (Ed.), Rearrangements in ground and excited states, 3rd edn. (pp. 91-129). Academic Press.

58. Jacobs, H. J. C., \& Laarhoven, W. H. (1995). Photochemistry of vitamin D and related compounds. In W. M. Horspool \& P.-O. Song (Eds.), Handbook of organic photochemistry and photobiology, 11th edn. (pp. 155-164). London: CRC Press.

59. Gauvin, A., Smadja, J., Aknin, M., Faure, R., \& Gaydou, E.-M. (2000). Isolation of bioactive $5 \alpha, 8 \alpha$-epidioxy sterols from the marine sponge Luffariella cf. variabilis. Canadian Journal of Chemistry, 78, 986-992. 